\title{
Global ICT Standardisation Forum for India (GISFI) and 5G Standardization
}

\author{
Ramjee Prasad \\ Founding Chairman GISFI \& Director of Center for TeleInfrastruktur \\ (CTIF), Aalborg University, Denmark; email: ramjee.prasad@gisfi.org, \\ prasad@es.aau.dk
}

Received July 2013; Accepted August 2013

\begin{abstract}
This paper covers a selected set of potentially high impact emerging technologies for $5 \mathrm{G}$ wireless communication as well as their current state of maturity and scenarios for the future. 'Interoperable', 'ubiquitous' and 'dynamic' are key words describing 5G networks and applications. The paper focuses on what are the necessary critical innovations, also undertaken within standardization and how to explore these as well as what are the expected challenges. It further identifies future directions for $5 \mathrm{G}$ and gives a vision of such communication system.
\end{abstract}

Keywords: Wireless innovative system for dynamically operating megacommunications (WISDOM), 5G, standardization.

\section{Introduction}

The International Telecommunication Union (ITU) [1] defines the standardization gap as disparities in the ability of developing countries, relative to developed ones, to access, implement, contribute to and influence international ICT standards and recommendations. In addition, for making international standards universally effective, it is important to identify and incorporate

Journal of ICT Standardization, Vol. 1, 123-136.

doi:10.13052/jicts2245-800X.12a1

(C) 2013 River Publishers. All rights reserved. 


\section{R. Prasad}

solutions for the country-specific scenarios. The Global ICT Standardisation Forum for India (GISFI) [2] was founded in 2009 as an Indian standardization body active in the area of Information and Communication Technologies (ICT) and related application areas, such as energy, telemedicine, wireless robotics, biotechnology in an effort to create a new coherence and strengthen the role of India in the world standardization process by mapping the achievements in ICT in India to the global standardization trends.

GISFI bases its work on the Indian Governmental policy and at the same time is cooperating with international standardization organizations to create internationally viable standards solutions that reflect the specifics of the Indian ICT scenario.

The Government of India direction for the Telecom growth in the country as specified in [3] states the Indian Telecom and ICT needs as related to cost-effective, energy-efficient and suitable for the Indian environment and user-specifics, technical products and solutions.

Figure 1 shows the standards evolution since the beginning of the wireless communication era till date. During the pioneer era, much fundamental research and development in the field of wireless communications took place. The cellular era started in 1980s with the launch of the analog cellular systems, followed by the digital wireless communication systems. Despite the large variety of existing communication systems, each development has been motivated by the same goal: to provide universal service facilities to the users. In the context of the Indian scenario, where despite the overall very high wireless penetration (862 million in January 2013 [4]), rural user density remains low due to the high deployment costs making services unaffordable by those users, the ubiquitous service provision is even more challenging.

Typically, $70 \%$ of the capital cost of cellular networks is in the access network as opposed to the backbone, which means that these networks depend on a certain user density for profitability. Hence, urban areas tend to be covered by multiple carriers, while rural areas typically have one or none.

When aiming to evolve the growth of the Tree of Standards (see Figure 1) to reach the 5G top, GISFI looks at a technology concept of a system, namely the Wireless Innovative System for Dynamically Operating MegaCommunications (WISDOM) [5, 6, 7] with the following key features:

- Combines established, competitive cellular standards with a promising frequency spectrum and novel enabling technologies;

- Reduced coverage, electricity and operational expenditure (OPEX) costs;

- Offers scalable and flexible technology options. 


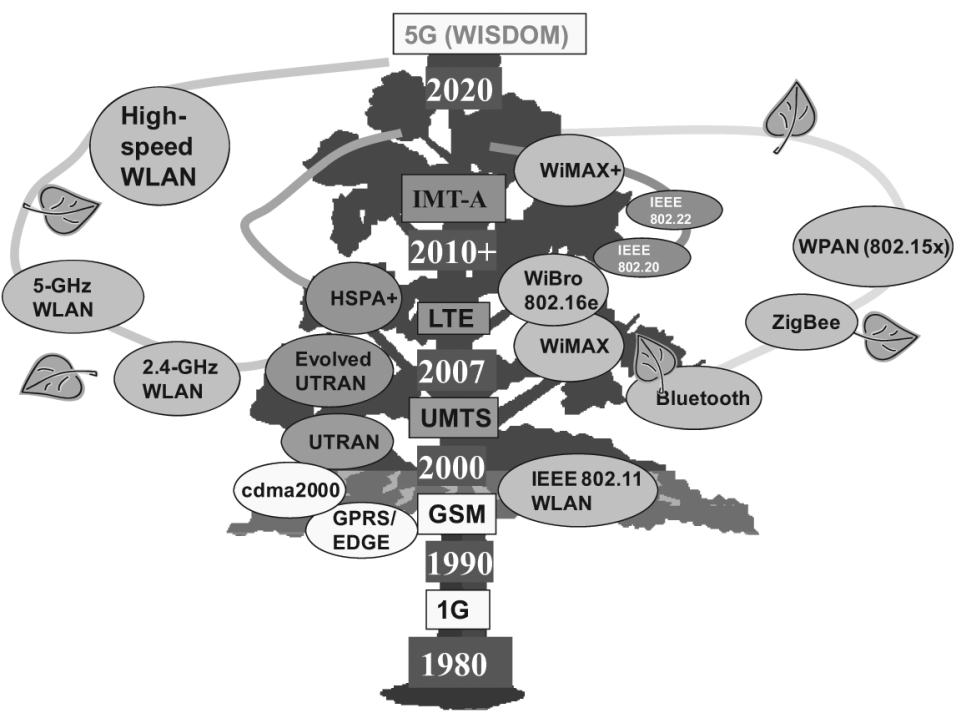

Figure 1 The tree of standards.

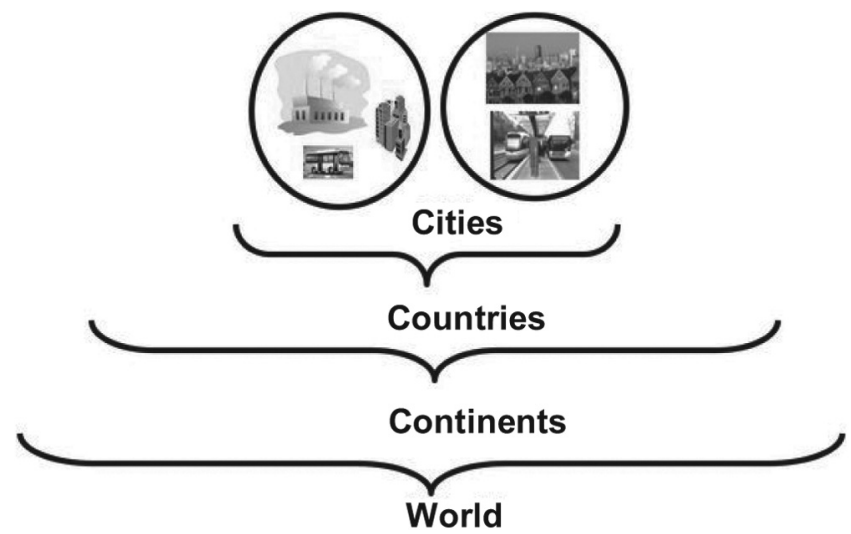

Figure 2 Global connectivity through WISDOM [5, 6, 7].

GISFI offers the vision of a future universally deployable wireless communication system that would make the pillar for enabling smart teleinfrastructures, which will offer services and applications of data rate more than tera/bit/second (Tbps), with a coverage extending from a city region, to a country, the continents, and the world to enable human-centric megacommunication applications. The WISDOM concept is shown in Figure 2. 
To achieve this vision, GISFI brings different major stakeholders together into its six Working Groups to define and specify the most suitable advanced technologies to lead to enabling 5G. The GISFI Working Groups are as follows [8]:

- Security and Privacy;

- Future Radio Networks;

- Internet of Things;

- Cloud and Service Oriented Networks;

- Green ICT;

- Spectrum;

- Special Interest Group.

This paper defines the steps undertaken within GISFI towards 5G standardization including the specifics of the Indian ICT scenario and offers a vision of a universally deployable $5 \mathrm{G}$ system.

The paper is organised as follows. Section 2 defines the scenarios and factors influencing the development, deployment and commercialization of 5G. The main research enablers are also outlined. Section 3 gives a vision of $5 \mathrm{G}$ and identifies some open research issues. Section 4 concludes the paper.

\section{Scenarios and factors Influencing Development, Deployment and Commercialization of 5G}

Possible scenarios for future wireless communications (e.g., 5G) based on an analysis of key uncertainties and long-term trends were described in [7].

It is envisioned that the need for high throughput will continue to increase in future wireless networks, driven by the rising needs of the mass market in the fields of bandwidth demanding applications such as: entertainment, multimedia, Intelligent Transport Systems (ITS), telemedicine, emergency and safety/security applications.

Futuristic applications such as: 3-dimensional (3D) Internet, virtual and augmented reality that combines data for all senses (audio, visual, haptic, digital scent), telehaptic applications like planet or deep sea exploration, networked virtual reality (e.g., video streaming from social networks - each user streams its own reality), and telepresence (e.g., immersive environments with applications in both the commercial and military fields), can push the demand for real-time, symmetric, wireless ubiquitous connectivity to an individual with a data rate well in excess of $250 \mathrm{Mbps}-$ a rate that far exceeds the capacity 
offered by current and emerging next generation wireless technologies and networking infrastructures.

At the same time, tremendous growth of the number of networked devices leading to the so-called trillion device network requires wireless solutions which are cost sensitive but also able to support mobility, since a large fraction of the devices will be associated with people and robots.

People's needs are changing too, including the needs to communicate in a very dynamic environment. In parallel, the demand for an efficient communication service, closer to the users, is rising. An efficient communication system is one able to follow the user wherever the user is, and able to adapt its traffic capabilities to the dynamic user environment.

In a first and most obvious direction, the development of future wireless communications will be determined by a very high public demand for wireless solutions and breakthroughs. A key challenge for reaching this scenario will be depending on whether technologies would deliver to match such high level of user expectations.

\subsection{Small Cell Deployment}

Small cell deployment has been identified as a means to dynamically bring the communication services closer to the user $[9,10]$. Small cells can be deployed where there is a temporary need, and areable to cover the traffic needs, which arise in an area for a limited time or may follow the users in their movement. And in many cases small cells are not owned by the network provider, but are directly in the ownership of the user or are part of the communications interface offered by a third party (e.g., a transport company). A user will not consider a small cell close if this small cell is not cost-efficient. The ability to be cost-efficient is coming from the capability to adapt to the user needs, to offer connectivity when and where the user wants it, to not waste expensive energy and use only the necessary resources (e.g. radio spectrum, capacity, etc). It must be cost-efficient by itself and must be used in a cost-efficient manner. Because the users' needs and habits vary from one moment to another, from a particular user to another, small cells must support heterogeneity and reconfigurability.

The main focus of the recent technical work with in GISFI (WG Future Radio Networks [8]) has focused on how to enable additional capacity, improved coverage, and energy/cost-efficient networks by the design, analysis, development and evaluation of procedures for fast network deployment and dynamic reconfiguration of small cells in various scenarios, also relevant to the Indian 
specifics. The processes needed to deploy small cells economically (how to deploy, where to deploy, how to deal with the increased number of small cell sites, etc.) are also being studied in order to propose new, economically viable mechanisms.

\subsection{Security}

The new age of wireless envisioned in the 1990s has finally come to pass. Remarkable achievements in wireless and other areas of telecommunications (e.g., ICT) have ushered in the new age of ubiquitous mobile communications. For such scenario to emerge successfully, wireless communication systems must deliver technological solutions that make it possible to eradicate or, at least, control the potentially dangerous aspects of this ubiquitous communication (e.g., related to security, trust and the protection of personal data; reliability and dependability); to extend longevity, boost economy and improve the quality of life.

For example, in the context of the small cell deployments, emerging vulnerabilities are the following:

- Devices can be compromised

- Can be turned into IMSI catcher

- User data can be eavesdropped/ modified

- Denial of Service attacks

- Call fraud

- Privacy issues

- Considerations of security for use of technologies use across operators.

In another scenario, the technological push will naturally lead into the new wireless communication era. A possible barrier to fulfill the technological promises bringing about long delays into technological adoption can be expected from obstacles posed by negative public sentiment, legal barriers and lack of funding.

Such opposition may come from lack of education or understanding of how technologies work, as well as legitimate safety and ethical concerns.

To this end, GISFI has undertaken work to perform a study [11] on the telecom security policy addressed to Indian wireless network operators and to network equipment manufacturers/ vendors (both to Indian or Global organizations). This is very much in line with the recommendations of Department of Telecom (DoT), Government of India (GoI), of April 2013, stating that "... the operators shall integrate only those network elements into their 
telecom networks which have been tested and certified from (Government) authorized and certified agencies/labs in India."

The greatest obstacles entail moral or ethical considerations involving the manipulation of personal and national security data related to wireless communications for critical infrastructures. Significant developments and breakthroughs in new technologies have produced raging success in the wireless and ICT field, but many of the most novel and effective solutions (e.g., cognitive radio networks; spectrum and infrastructure sharing) are slow to appeal with consumers, government agencies, advocacy groups and in some cases, entire regions of the world.

\subsection{Green ICT}

The ICT industry is a contributor to the phenomenon of global warming, with a current footprint of $3 \%$ of the consumed energy. However the energy consumption of ICT is rising at a rate of $16-20 \%$ per year. It is also shown that $90 \%$ of the global cellular network power consumption belongs to the network side and, of that, $97 \%$ is consumed by the Radio Access Network [12]. Only recently has the idea of energy efficiency come to the attention of researchers, authorities and mobile operators. In the past, mobile networks had been designed to optimize spectral efficiency or capacity, but not energy efficiency. Good energy and spectral efficiency is translated into economics gain, because more traffic is sent over the network, traffic that generates revenue. Value added services like multimedia services and entertainment services increases the revenue that can be generated from the spectrum. Another efficient use of the spectrum is to "borrow" the unutilised spectrum a primary operator has to a secondary one as long as the primary user does not need it. This approach is another good revenue opportunity for primary operators of the spectrum.

In order to obtain any degree of energy saving, the attention should focus on the radio access side of the cellular network. Then the challenge is to design flexible techniques and algorithms in order to reduce the base station power consumption. Authors in [13] have identified two ways to reduce the energy consumption of Base Stations: (1) by having energy-aware components in BSs and (2) by energy-aware deployment strategies of BSs. However, the paper does not elaborate on those strategies.

Within GISFI, activities span energy efficiency issues related to greening of the telecom infrastructure as well as related to more energy-efficient utilization of the spectrum. These activities are undertaken within the corresponding Working Groups on Green ICT and Spectrum. 
In India, TRAI [4] recommends that all telecom products, equipments and services in the telecom network should be energy and performance assessed and certified with a 'Green Passport' utilizing the Energy consumption Rating (ECR) and energy 'passport' determined by the year 2015. However, at this point of time telecom services have been excluded from the scope of the proposed 'Green Passport'. Telecom equipments are the current subject of focus, since adoption of energy-efficient telecom equipments would lead to overall energy efficiency of network and services [14]. Further, the ICT infrastructure used in telecommunication core networks also needs to be certified for energy-efficient operations. GISFI provides a generic definition of telecommunications equipment efficiency as the ratio of power to the useful work done[15]. Useful work and power are terms that should be defined in each supplemental standard. Additionally, in the GISFI supplemental standards, the following would be defined:

a. The scale of the energy efficiency metric for each class of equipment so that the metrics for similar class of equipment may be made comparable.

b. The lower the value of the energy efficiency metric, the more energy efficient is the metric compared to others of similar configuration.

GISFI has produced three technical reports (TRs) on Green ICT till date, the most relevant of which are the TR on Metrics and Measurement Methods for Energy Efficiency (GISFI TR GICT.105 [8]) and the draft technical specification (TS) on the Metrics and Measurement Methods for Energy Efficiency: General Requirements. GISFI's approach is not to reinvent the wheel, but re-use existing technical specifications from international standards wherever applicable and develop specifications if a clear technical gap is identified in the Indian context. As part of its efforts, GISFI has produced the following:

- Classification and prioritization of telecom equipments;

- Survey of International GICT standards and best practices;

- Recommended international standards with Indian requirements;

- Recommended GICT metrics and methods for measurement;

- Recommended assessment procedure and scales for rating.

\subsection{Spectrum}

In general, mobile services can use spectrum upto $6 \mathrm{GHz}$ due to requirements of building penetration, multi-path, non line-of-sight, etc.

Hence, globally, spectrum managers are moving the existing line-of-sight, fixed links in frequency bands below $6 \mathrm{GHz}$, to higher frequency bands or alternate physical media like OFC, etc. In general, radio use in higher frequencies 
is a lot lighter, and currently, this gets a lot of attention as a potential way to realize $5 \mathrm{G}$ technologies.

In order to create a communication system that is revenue-rich, the $5 \mathrm{G}$ technologies must be realized so that they enable human-centric value-added services and applications. This will highly depend on cellular machine-tomachine (M2M) connectivity. Use of the millimeter wave communication links can add to the capacity enabled by small cells, leading to a new type of cellular architecture where base station connectivity to devices or other base stations can be based on use of millimetre wave links [16]. Still, the high absorption rate of the millimeter wave electromagnetic signal $(60 \mathrm{GHz}$ and beyond, like the e-band at the $70 \mathrm{GHz}$ for industrial applications, but also higher bands such as $140 \mathrm{GHz}$ and $220 \mathrm{GHz}$ ) poses great challenges for their utilization in non - line of sight (LOS) and mobile connections. On the other hand the high directionality attained in this band can be used to increase spatial multiplexing.

White spaces are being demanded and proposed to be used for low power un-licensed, Wi-Fi type usages, along with use of cognitive radio.

\subsection{The Human-Centric Paradigm}

In order to fulfil the needs of the modern user, 5G should offer human-centric connectivitywith data rates in the Tbps range and a coverage extending from a city region, to a country, the continents, and the world. The human-centric aspect will enable that connection traffic and application needs will grow to demand in turn $5 \mathrm{G}$ connectivity, which will facilitate deployment of $5 \mathrm{G}$ technologies and equipment.

Today, India is the second largest country in terms of mobile user base next to China[17]. Internationally, India is also considered the most potential market in mobile. It has been predicted that India will become the world's third largest smartphone market by 2017 after China and U.S [17] based on the 67 million smartphone subscribers at the time of the report, which is $6 \%$ of the total subscribers in India, growing at the rate of 52\% YoY.

Within GISFI the human-centric paradigm and required connectivity are reflected within the work of the Working Groups on Internet of Things (IoT) and Cloud and Service Oriented Networks (CSoN).

The WG IoT has defined several use cases to help define the technical and usage requirements for human-centric connectivity and applications. 5G should seamlessly bridge the virtual and physical worlds offering the same level of all-senses, context-based, rich communication experience over fixed 


\section{R. Prasad}

and wireless networks to users. As a first objective in reaching this vision, there should be no limits on wireless data rates. In a human-centric 5G scenario, each user should be provided with ubiquitous personalized network access at very high sustainable data rates approaching the Ethernet current state-ofthe-art (10+ Gbps). A ubiquitous and pervasive wireless network offering a sustainable 10 Gbps bit rate (reaching up to 1 Tbps in a bursty mode) can be used as an alternative to Ethernet, and an access network to Tbps fiber networks.

Because $5 \mathrm{G}$ will be a plethora of interworking technologies governed by separate specifications, it is important to find technology solutions and standardize the interconnectivity for end to end telecom service provisioning across technologies and operators. Part of the activities within the WG on CSoN, have focused on this challenge [8].

The challenges related to the realization of the $5 \mathrm{G}$ end-to-end scenario are concerning the architecture design; the wired network segment transport protocol and the wireless/wireline functional interface nodes.

An optimized end-to-end architecture should be designed with the aim to provide excellent system performance in terms of bandwidth, utilization of network resources, reconfiguration capability, quality of service $(\mathrm{QoS})$ and cost efficiency as well as survivability and security. Use of the cloud computing benefits and their integration is crucial in the context of the enormous volume of bursty traffic generated by the $5 \mathrm{G}$ solution.

In order for a cloud system to support the large flow of real-time data for human-centric 5G applications, the following general cloud requirements can be identified.

- Related to data rates and latency: The data should be pushed out to the cloud, and then pushed to the user because polling requires too much time and uses too much bandwidth. Further, the data needs to flow quickly and effortlessly through the system, through a real-time database, which means that it should be able to stay in its simplest format.

- Related to security: the 5G application should be able to stream its data into the cloud without exposing its data to the dangers of communicating it over the Internet. Further, support of different user types accessing a single service should also be enabled. Resource configurations should also be performed in a secure way for each service across multiple cloud infrastructures.

- Related to semantics: the information provided by different sensors or other $5 \mathrm{G}$ devices is usually in a specific format allowing it only to be used 
by a proprietary application or to be controlled only by specific systems. But the provided information could be used by many other applications. It is therefore necessary for open interfaces and data formats to allow for using the different sensor outputs in applications, programming etc.

- Related to mobility: Specific and smart mechanisms should exist for controlling and exploiting the mobility of real-world entities and attached $5 \mathrm{G}$ devices.

\section{A Vision of $5 G$}

Networks will be the single, most indispensable element of future wireless connectivity, building an infrastructure of large-scale, complex and highly networked systems whose efficiency, sustainability and protection would require intelligent, interoperable and secure ICT solutions and novel business models. Users are not only humans, but also machines. At the core of $5 \mathrm{G}$ applications are machine-to-machine (M2M) communications enabled by technological breakthroughs in a plethora of scientific areas, wireless and wired communications, artificial intelligence, Internet, robotics, space, and so forth. This interworked 5G technological landscape is shown in Figure 3.

Always-on capacity, connectivity, and pervasiveness are key characteristics of the future $5 \mathrm{G}$ communication scenario that drive the emergence of new environments that evolve from the gradual development and combination of present day cellular communications and related advances (e.g., small cells), Internet of Things (IoT), M2M, and cloud computing, and towards a more advanced vision of fully reprogrammable mobile devices, able to communicate with each other autonomously based on a given event context and part of a scale-free self-organized communication systems.

There are three main aspects to realizing $5 \mathrm{G}$ communication systems.

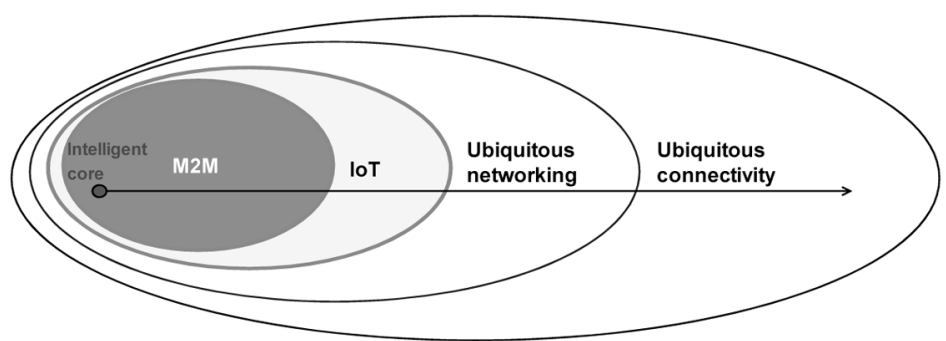

Figure 3 Plethora of technologies to deliver 5G services and applications. 
- Enabling the 5G intelligent core. M2M and IoT are key for realizing the intelligent core, which in turn is key for enabling seamless ubiquitous networking and connectivity in a $5 \mathrm{G}$ context. In relation to the specifics of the Indian scenario, this intelligent core will be a turning point for bringing mobile services also to the Indian rural areas [18]. M2M and IoT are the key enabling technologies for a pervasive and always-connected 5G mobile services. Research challenges to fully deployable intelligent core are related but not limited to handling the big data collected through M2M and IoT communications (e.g., heterogeneous gateways, energy efficiency, decentralization of routing, naming and addressing), and to security, privacy and trust.

- Enabling ubiquitous connectivity. This feature has two aspects. On one hand, technical challenges relate to sufficient coverage range even in a scenario of very high mobility and data rates, and on the other, to moving application from device to device without any content interruption. Use of millimeter wave links as discussed previously, novel multiple antenna concepts, virtualization, small cell deployments, novel spectrum usage methods, are some of the key research enabling areas for ubiquitous connectivity.

- Enabling ubiquitous networking. This means that regardless of how many access networks are integrated for connectivity purposes, the quality of the delivered service must be retained end-to-end. In view of increased importance of the cloud computing concept for supporting the big data originating from the intelligent $5 \mathrm{G}$ core, end-to-end ubiquitous networking will require interoperable decentralized service-oriented mechanisms with support of real-time interactions. And end-to-end ubiquitous networking architecture will blend concepts and techniques from the three main $5 \mathrm{G}$ elements (see Figure 3), for example:

- Service descriptions and utility measurement specifications for intelligent core services, which will be executed over cloud infrastructures.

- Scheduling and resource management techniques that take into account shared (multi-tenant) access to (heterogeneous locationdependent) intelligent core resources, in addition to conventional (location-independent) computing resources.

- Security and access controls mechanisms that take into account the sharing of resources (e.g., physical and virtual). 


\section{Conclusions}

$5 \mathrm{G}$ is a concept where personalization meets connectivity and networking technological innovations by integrating under one interoperable umbrella leading technologies, such as advanced M2M communication technologies, cooperative and cognitive radio and networking technologies, data mining and decision-making technologies, security and privacy protection technologies, cloud computing technologies, with advanced sensing and actuating technologies. $5 \mathrm{G}$ bundles multi-radio, multi-band air interfaces to support portability and nomadicity in ultra-high data-rate communications using novel concepts and cognitive technologies.

GISFI is currently undertaking $5 \mathrm{G}$ standardization by defining recommendations for the realization of the WISDOM concept.

\section{References}

[1] International Telecommunication Union (ITU), www.itu.int.

[2] Global ICT Standardisation Forum for India (GISFI), www.gisfi.org.

[3] Government of India, Department of Telecommunicaitons, "National Telecom Policy 2012," http://www.dot.gov.in/telecom-polices/national-telecom-policy-2012

[4] Telecom Regulatory Authority of India (TRAI), "'Highlights on Mobile Data Subscription in India as of January 2013," Press Release Report, New Delhi, March 2013, www.trai.gov.in.

[5] C.-I.Badoi, N. Prasad, V.Croitoru and R. Prasad, "5G Based on Cognitive Radio,"Springer International Journal on Wireless Personal Communications (2011) 57:441-464. DOI 10.1007/s 11277-010-0082-9.

[6] R. Prasad, "Future Networks and Technologies Supporting Innovative Communications," in Proceedings of IEEE IC-NIDC2012, Bejing, China, September 2012.

[7] R. Prasad, "High Impact Emerging Technologies for Beyond 2020 Wireless Communications," Proceedings of WWRF, October 2012, Berlin, Germany.

[8] Global ICT Standardisation Forum for India, GISFI, www.gisfi.org.

[9] 3GPP TR 36.814 V.9.0.0. (2010-03), "Further Advancements for E-UTRA physical layer aspects," Release 9.

[10] 3GPP TS 32.101 V11.1.0 (2012-12), "Telecommunication management; Principles and high level requirements", Release 11.

[11] WG Security, GISFI_SP_201303376, “(Telecom Security) Policy Study and Proposals," www.gisfi.org, March 2013.

[12] E.C. Strinati and L. Herault, "Holistic approach for future energy efficient cellular networks," Elektrotechnik \& Informationstechnik, vol. 127, Nov. 2010, pp. 314320.

[13] Arnold, O.; Richter, F.; Fettweis, G.; Blume, O., "Power consumption modelling of different base station types in heterogeneous cellular networks," Future Network and Mobile Summit, 2010 , vol., no., pp.1,8, 16-18 June 2010. 
[14] WG Green ICT, GISFI_GICT_201301389, “Approach towards Implementation of Green Telecom in India,"www.gisfi.org, January 2013.

[15] WG Green ICT,GISFI_GICT_201301357, "Metrics and Measurement Methods for Energy Efficiency: General Requirements,” www.gisfi.org, December 2012.

[16] Millimeter-Wave Transceiver Technologies for 5G Cellular Networks - http:// global.samsungtomorrow.com/?p=24093\#sthash.x3KTbC9y.dpuf.

[17] Indian Mobile Landscape 2013, http://www.iamwire.com/2013/06/indian-mobile-land scape-2013/, June 2013.

[18] Confederation of Indian Industry-CII, "Machine-to-Machine: Vision 2020 Is India ready to seize a USD 4.5 trillion M2M opportunity?", TeleTech 2013 report, www. deloitte.com/in

\section{Biography}

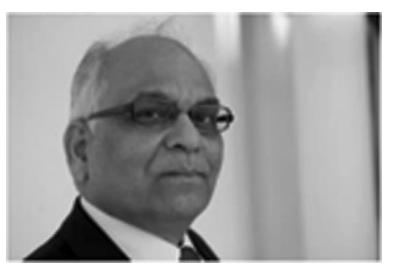

Ramjee Prasad is currently the Director of the Center for TeleInfrastruktur (CTIF) at Aalborg University, Denmark and Professor, Wireless Information Multimedia Communication Chair.

Ramjee Prasad is the Founding Chairman of the Global ICT Standardisation Forum for India (GISFI: www.gisfi.org) established in 2009. GISFI has the purpose of increasing of the collaboration between European, Indian, Japanese, North-American and other worldwide standardization activities in the area of Information and Communication Technology (ICT) and related application areas. He was the Founding Chairman of the HERMES Partnership - a network of leading independent European research centres established in 1997, of which he is now the Honorary Chair.

$\mathrm{He}$ is the founding editor-in-chief of the Springer International Journal on Wireless Personal Communications. $\mathrm{He}$ is a member of the editorial board of other renowned international journals including those of River Publishers. Ramjee Prasad is a member of the Steering, Advisory, and Technical Program committees of many renowned annual international conferences including Wireless Personal Multimedia Communications Symposium (WPMC) and Wireless VITAE. He is a Fellow of the Institute of Electrical and Electronic Engineers (IEEE), USA, the Institution of Electronics and Telecommunications Engineers (IETE), India, the Institution of Engineering and Technology (IET), UK, and a member of the Netherlands Electronics and Radio Society (NERG), and the Danish Engineering Society (IDA). He is a Knight ("Ridder") of the Order of Dannebrog (2010), a distinguished award by the Queen of Denmark. 